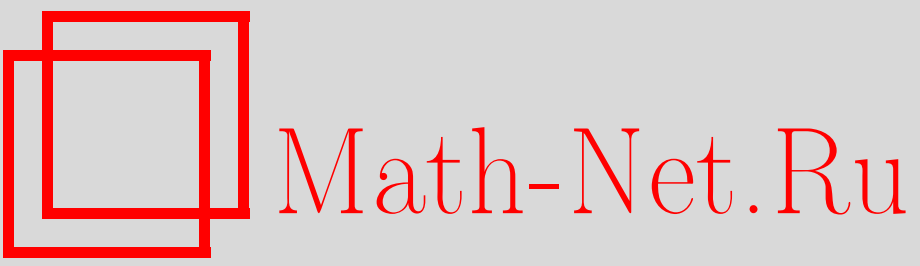

А. В. Шутов, Фракталы Рози и их теоретико-числовые приложения, Итоги науки и техн. Сер. Соврем. мат. и ее прил. Темат. обз., 2019, том 166, 110119

DOI: https://doi.org/10.36535/0233-6723-2019-166-110-119

Использование Общероссийского математического портала Math-Net.Ru подразумевает, что вы прочитали и согласны с пользовательским соглашением http://www . mathnet.ru/rus/agreement

Параметры загрузки:

IP : 54.157 .27 .8

26 апреля 2023 г., $13: 32: 29$ 


\title{
ФРАКТАЛЫ РОЗИ И ИХ ТЕОРЕТИКО-ЧИСЛОВЫЕ ПРИЛОЖЕНИЯ
}

\author{
(c) 2019 г. $\quad$ A. B. ШУтОВ
}

\begin{abstract}
АннотАция. В работе построены и изучены разбиения Рози порядка $n$ для некоторого класса чисел Пизо. Данные разбиения представляют собой разбиения тора на фрактальные множества. При этом действие некоторого сдвига тора на введенных разбиениях сводится к перекладыванию тайлов разбиений. Получен ряд приложений введенных разбиений к изучению соответствующего сдвига тора. В частности, показано, что тайлы разбиения оказываются множествами ограниченного остатка относительно рассматриваемого сдвига. Кроме того, получен ряд приложений к изучению множеств натуральных чисел, имеющих заданное окончание жадного разложения по линейной рекуррентной последовательности, и к обобщенным круговым умножениям КнутаМатиясевича.
\end{abstract}

Ключевые слова: разбиение Рози, система счисления, множество ограниченного остатка, аддитивная задача.

\section{RAUZY FRACTALS AND THEIR NUMBER-THEORETIC APPLICATIONS}

\section{(c) 2019 A. V. SHUTOV}

\begin{abstract}
In this paper, we construct and study Rauzy partitions of order $n$ for a certain class of Pisot numbers. These partitions are partitions of a torus into fractal sets. Moreover, the action of a certain shift of the torus on partitions introduced is reduced to rearranging the partition tiles. We obtain a number of applications of partitions introduced to the study of the corresponding shift of the torus. In particular, we prove that partition tiles are bounded-remainder sets with respect to the shift considered. In addition, we obtain a number of applications to the study of sets of positive integers that have a given ending of the greedy expansion by a linear recurrent sequence and to generalized Knuth-Matiyasevich multiplications.
\end{abstract}

Keywords and phrases: Rauzy partition, numeral system, bounded remainder set, additive problem. AMS Subject Classification: $11 \mathrm{~J} 71,11 \mathrm{Kxx}, 28 \mathrm{~A} 80$

1. Введение. В 1982 г. французский математик Жерар Рози в [23] ввел множество с фрактальными границами, связанное со сдвигом тора $x \rightarrow x+\left(\zeta^{-1}, \zeta^{-2}\right) \bmod \mathbb{Z}^{2}$, где $\zeta$ - корень кубического уравнения $\zeta^{3}-\zeta^{2}-\zeta-1=0$, и применил этот объект к построению нового примера множеств ограниченного остатка относительно данного сдвига тора. Введенное множество получило впоследствии название фрактала Рози. Фрактал Рози был позднее применен к построению некоторого бесконечного слова с функцией сложности $2 n+1$, кодирующего данный сдвиг тора и ставшего источником новых примеров и задач в комбинаторике слов.

В дальнейшем конструкция фрактала Рози многократно обобщалась и находила новые применения в комбинаторике слов, теории динамических систем, дискретной геометрии и других разделах математики. Подробности и дальнейшие ссылки могут быть найдены в $[15,22,24]$. При этом теоретико-числовые свойства фракталов Рози постепенно практически выпали из рассмотрения. 
В 2005 г. В. Г. Журавлев на основе классического фрактала Рози построил бесконечную последовательность разбиений тора, названную им разбиениями Рози, и показал, что все тайлы этих разбиений также являются множествами ограниченного остатка.

Настоящая работа представляет собой обобщение конструкции разбиений Рози на случай очень широко класса фракталов Рози, связанных с числами Пизо. Данное обобщение позволило получить аналог теоремы Журавлева о множествах ограниченого остатка, а также ряд других приложений, связанных с теоретико-числовыми свойствами сдвига тора, ассоциированного с фракталом Рози. Кроме того, удалось получить совершенно новый класс приложений, связанных с теоретико-числовыми свойствами множеств натуральных чисел, имеющих заданное окончание жадного разложения по некоторым линейным рекуррентным последовательностям.

Данная работа представляет собой расширенную версию доклада, прочитанного автором на IV Международной конференции «Актуальные проблемы прикладной математики» (22-26 мая 2018 г.).

2. Фракталы Рози. Можно выделить 3 основных подхода к определению фракталов Рози:

(1) дискретизация прямой (см. [22, гл. 7]);

(2) теория геометрических подстановок (см. [14] и [22, гл. 8]);

(3) $\beta$-разложения, связанные с числами Пизо (см. [11]).

Мы будем использовать третий подход как наиболее близкий к теории чисел.

Пусть $\beta$-число Пизо степени $d, \beta^{(1)}, \ldots, \beta^{\left(r_{1}\right)}$ - действительные сопряженные, $\beta^{\left(r_{1}+1\right)}$, $\overline{\beta^{\left(r_{1}+1\right)}}, \ldots, \beta^{\left(r_{1}+r_{2}\right)}, \overline{\beta^{\left(r_{1}+r_{2}\right)}}$ - комплексные сопряженные. Ясно, что $r_{1}+2 r_{2}=d-1$. Кроме того, из определения числа Пизо вытекает, что $|\beta|>1,\left|\beta^{(k)}\right|<1$.

Для произвольного действительного числа $x$ можно определить жадное разложение по степеням $\beta$ :

$$
x=\sum_{k=k(x)}^{m(x)} \varepsilon_{k}(x) \beta^{k},
$$

где $k(x) \geqslant-\infty, m(x)<\infty$ и

$$
0 \leqslant x-\sum_{k=m_{1}}^{m(x)} \varepsilon_{k}(x) \beta^{k}<\beta^{m_{1}}
$$

при $-\infty<m_{1}<m(x)$. Определим отображение

$$
\Phi(x)=\left(x^{(1)}, \ldots, x^{\left(r_{1}\right)}, \operatorname{Re} x^{\left(r_{1}+1\right)}, \operatorname{Im} x^{\left(r_{1}+1\right)}, \ldots, \operatorname{Re} x^{\left(r_{1}+r_{2}\right)}, \operatorname{Im} x^{\left(r_{1}+r_{2}\right)}\right),
$$

где

Множество

$$
x^{(j)}=\sum_{k=k(x)}^{m(x)} \varepsilon_{k}(x)\left(\beta^{(j)}\right)^{k} .
$$

$$
\mathcal{T}=\overline{\{\Phi(x): k(x) \geqslant 0\}}
$$

называется фракталом Рози, связанным с иррациональностью $\beta$.

Геометрия фрактала Рози тесно связана с арифметикой $\beta$-разложений. Приведем только один результат (см. [12]).

Теорема 1. $\beta$-Разложение любого числа $x \in \mathbb{Q}(\beta)$ конечно тогда и только тогда, когда 0 является внутренней точкой фрактала Рози $\mathcal{T}$.

Вообще говоря, фракталы Рози не обязаны быть связными множествами и тем более не обязаны быть связанными со сдвигом тора. Как минимум, требуется, чтобы $\mathcal{T}$ представляло собой развертку $d$-мерного тора. Другими словами, требуется, чтобы существовала $d$-мерная решетка $L$, для которой $\mathcal{T}$ является фундаментальной областью, т.е.

(1) для любой точки $x \in \mathbb{R}^{d}$ существует такая точка $x^{\prime} \in \mathcal{T}$, что $x \equiv x^{\prime}(\bmod L)$;

(2) любые две точки $x, x^{\prime} \in \mathcal{T}$, не лежащие на границе $\mathcal{T}$, не сравнимы по модулю решетки $L$. 
Теорема 2. Пусть $\beta$ является корнем уравнения $x^{d}-t_{1} x^{d-1}-\ldots-t_{d}$. Фрактал Рози $\mathcal{T}$ является разверткой $d$-мерного тора тогда и только тогда, когда

$$
t_{1} \geqslant t_{2} \geqslant \ldots \geqslant t_{d-1} \geqslant t_{d}=1 .
$$

Доказательство теоремы 2 получается применением к критерию из [13] одного результата из [18]. Теорема 2 позволяет определить каноническую проекцию $\pi$ из множества $\mathcal{T}$ в тор $\mathbb{R}^{d} / L$.

Далее всюду будем считать, что условия теоремы 2 выполнены.

3. Разбиения Рози. В случае классического фрактала Рози $\left(d=3\right.$ и $\left.t_{1}=t_{2}=1\right)$ существует каноническое разбиение (см. [23])

$$
\mathcal{T}=\mathcal{R} \sqcup \mathcal{G} \sqcup \mathcal{B}
$$

на три подобных ему фрактала. Разбиение (1) определяет перекладывание $S$ областей $\mathcal{R}, \mathcal{G}$ и $\mathcal{B}$, которое оказывается изоморфным сдвигу тора. При этом подобие множеств $\mathcal{R}, \mathcal{G}$ и $\mathcal{B}$ исходному фракталу Рози $\mathcal{T}$ позволяет проитерировать разбиение $(1)$ классическим методом инфляциидефляции и получить бесконечную последовательность разбиений (см. [3]). Однако обобщение этого подхода на произвольные фракталы Рози наталкивается на принципиальные трудности, связанные с тем, что общее множество $\mathcal{T}$ не разбивается на подобные ему множества. Преодоление этих трудностей требует двух новых определений фрактала Рози. Одно из этих определений основано на теории конечных автоматов, второе - на использовании линейных рекуррентных последовательностей.

Слово называется допустимым, если оно распознается конечным автоматом

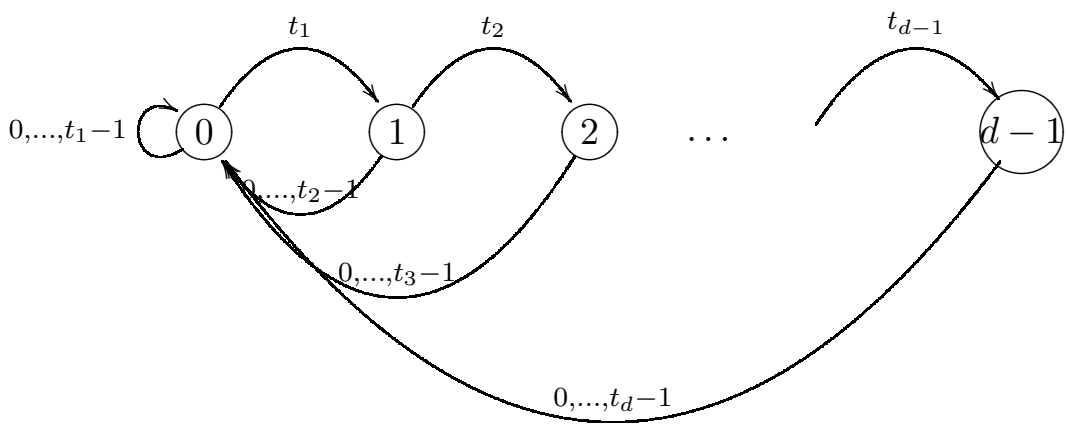

Определение допустимых слов мотивировано следующим результатом (см. [11]).

Предложение 1. Разложение $x=\sum_{k=k(x)}^{m(x)} \varepsilon_{k}(x) \beta^{k}$ является жадным тогда и только тогда, когда слово $\varepsilon_{m(x)} \varepsilon_{m(x)-1} \ldots \varepsilon_{k(x)}$ допустимо.

Пусть $\mathrm{Adm}$ - множество всех допустимых слов, $\mathrm{Adm}_{k}$ - множество допустимых слов длины $k$ и $T_{k}=\sharp \mathrm{Adm}_{k}$. Оказывается, что $T_{k}$ является линейной рекуррентной последовательностью, связанной с $\beta$.

\section{Предложение 2.}

$$
T_{k}=t_{1} T_{k-1}+\ldots+t_{d-1} T_{k-d+1}+T_{k-d}
$$

Любое целое неотрицательное $N$ допускает жадное $T$-разложение

$$
N=\sum_{k=0}^{m(N)} \varepsilon_{k}(N) T_{k}
$$

по последовательности $\left(T_{k}\right)$. Жадность разложения $(2)$ означает, что для любого $m_{1}<m(N)$ выполняются неравенства

$$
0 \leqslant N-\sum_{k=m_{1}}^{m(N)} \varepsilon_{k}(N) T_{k}<T_{m_{1}} .
$$


Введем отображения $\Phi_{\mathrm{Adm}}$ и $\Phi_{\mathbb{N}}$, заданные формулами

$$
\Phi_{\mathrm{Adm}}(a)=\Phi\left(\sum_{k=0}^{m} a_{k} \beta^{k}\right), \quad \Phi_{\mathbb{N}}(N)=\Phi\left(\sum_{k=0}^{m(N)} \varepsilon_{k}(N) \beta^{k}\right) .
$$

Здесь $a=a_{0} a_{1} \ldots a_{m} \in \mathrm{Adm}$ - допустимое слово, $N \in \mathbb{N}$. Из определений вытекает следующая теорема.

Теорема 3. Справедливы равенства

$$
\mathcal{T}=\overline{\Phi_{\operatorname{Adm}}(\operatorname{Adm})}=\overline{\Phi_{\mathbb{N}}(\mathbb{N})} .
$$

Пусть $\operatorname{Adm}(j)$ - множество допустимых слов, для которых пути в графе заканчиваются в вершине $j$.

Предложение 3. Имеет место разбиение

$$
\mathcal{T}=\mathcal{R}_{0} \sqcup \ldots \sqcup \mathcal{R}_{d-1},
$$

где

$$
\mathcal{R}_{j}=\overline{\Phi_{\operatorname{Adm}}(\operatorname{Adm}(j))} .
$$

Разбиение (3) называется разбиением Рози порядка 0.

Определеним перекладывание $S$ областей $\mathcal{R}_{j}$ равенством $S(x)=x+\Phi\left(T_{\beta}^{j}(1)\right)$, если $x \in \mathcal{R}_{j}$, где $T_{\beta}(x)=\{\beta x\}$. Определим также отображение сдвига

$$
R_{\beta}: \mathbb{T}^{d-1} \rightarrow \mathbb{T}^{d-1}, \quad\left(x_{1}, \ldots, x_{d-1}\right) \rightarrow\left(\left\{x_{1}+\beta^{-1}\right\}, \ldots,\left\{x_{d-1}+\beta^{-d+1}\right\}\right),
$$

отображение $S_{\mathrm{Adm}}: \mathrm{Adm} \rightarrow \mathrm{Adm}$, заключающееся в переходе к лексикографически следующему допустимому слову, и отображение

$$
S_{\mathbb{N}}: \mathbb{N} \rightarrow \mathbb{N}, \quad S_{\mathbb{N}}(n)=n+1 .
$$

Теорема 4. Имеет место коммутативная диаграмма

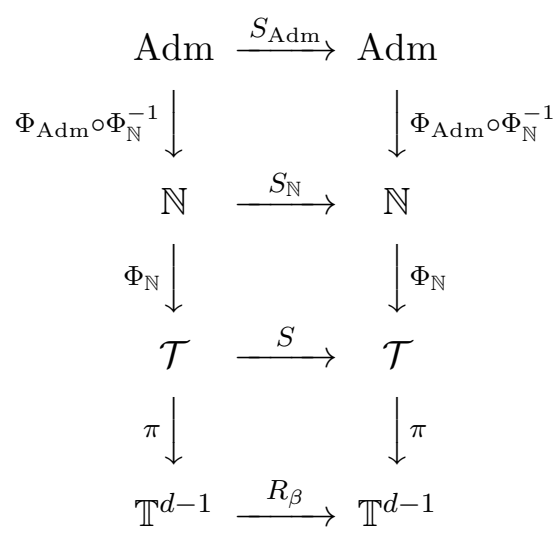

Теорема 4 позволяет переформулировать результаты о действии перекладывания $S$ на фрактале Рози $\mathcal{T}$ в результаты о действии сдвига $R_{\beta}$ на $(d-1)$-мерном торе и обратно.

Перейдем теперь к определению разбиения Рози произвольного порядка. Выберем некоторое слово $u \in \operatorname{Adm}_{d-1}(j)$. Пусть $\widetilde{A}_{m}(j)-$ множество слов $w$ длины $m$, для которых $u w \in \operatorname{Adm}$. Легко проверить, что определение множества $\widetilde{A}_{m}(j)$ не зависит от выбора $u$. Пусть $w \in \widetilde{A}_{m}(j)$. Для $u \in \operatorname{Adm}$ обозначим через $\operatorname{Adm}(u)$ множество допустимых слов, заканчивающихся на слово $u$. Рассмотрим множества

$$
\mathcal{R}_{m, j}(w)=\bigsqcup_{u \in \operatorname{Adm}_{d-1}(j)} \overline{\Phi_{\operatorname{Adm}}(\operatorname{Adm}(u w))} .
$$


Теорема 5. Имеет место разбиение

$$
\mathcal{T}=\bigsqcup_{j=0}^{d-1} \bigsqcup_{w \in \widetilde{A}_{m}(j)} \mathcal{R}_{m, j}(w)
$$

Разбиение (4) называется разбиением Рози порядка $m$.

4. Иррациональные сдвиги тора. Перейдем теперь к описанию приложений разбиений Рози к изучению иррационального сдвига тора $R_{\beta}$. В силу теоремы 4 результаты можно формулировать в терминах действия перекладывания $S$ на фрактале Рози $\mathcal{T}$. Мы рассмотрим три типа таких приложений: построение множеств ограниченного остатка, задачу об отображениях первого возвращения для сдвига тора и задачу о времени первого попадания последовательности дробных долей линейной функции в область.

Действие перекладывания $S$ на разбиении (4) описывается следующими теоремами.

Теорема 6. Пусть $w_{\max }(m, j)$-лексикографически максимальное слово из $\widetilde{A}_{m}(j)$. Тогда для любого слова $w \in \widetilde{A}_{m}(j), w \neq w_{\max }(m, j)$ найдется такое слово $w^{\prime} \in \widetilde{A}_{m}(j)$, что

$$
S\left(\mathcal{R}_{m, j}(w)\right)=\mathcal{R}_{m, j}\left(w^{\prime}\right) .
$$

Теорема 7. Справедливо равенство

$$
\bigsqcup_{j=0}^{d-1} S\left(\mathcal{R}_{m, j}\left(w_{\max }(m, j)\right)\right)=\bigsqcup_{j=0}^{d-1} \mathcal{R}_{m, j}(\underbrace{0 \ldots 0}_{m}) .
$$

Отметим, что разбиения, удовлетворяющие заключениям теорем 6, 7 впервые появились в [6] при изучении множеств ограниченного остатка.

Выберем некоторую область $X \in \mathcal{T}$ и рассмотрим функцию

$$
N(X, n)=\sharp\left\{k: 0 \leqslant k \leqslant n-1, S^{k}(0) \in X\right\} .
$$

Из теоремы 4 , иррациональности сдвига тора $R_{\beta}$ и знаменитой теоремы Вейля о равномерном распределении вытекает, что последовательность $\left\{S^{k}(0)\right\}$ равномерно распределена на фрактале Рози $\mathcal{T}$, т.е.

$$
N(X, n)=n|X|+o(n)
$$

Вопрос о возможности улучшения остаточного члена формулы (5) в общем случае до настоящего времени остается нерешенным.

Множество $X$ называется множеством ограниченного остатка, если остаточный член $r(X, n)=$ $N(X, n)-n|X|$ асимптотической формулы (5) имеет порядок $O(1)$, т.е. существует такая не зависящая от $n$ постоянная $C$, что

$$
|N(X, n)-n| X|| \leqslant C
$$

Множества ограниченного остатка впервые были введены Гекке (см. [19]). В настоящее время им посвящено огромное количество работ, из которых мы отметим $[3,5,6,17,20,23]$.

Теорема 8. Все множества $\mathcal{R}_{m, j}(w)$ являются множествами ограниченного остатка. Константа $C$ может быть выбрана не зависящей от $w$ и $\mathrm{m}$.

Теорема 8 может быть выведена из теорем 6 и 7 методами работы [6]. Можно также дать альтернативное доказательство теоремы 8, основываясь на аккуратном подсчете слов из $\mathrm{Adm}(u w)$ с использованием асимптотических формул для последовательности $\left\{T_{k}\right\}$. Отметим также, что теорема 8 автоматически порождает примеры множеств ограниченного остатка относительно сдвига $R_{\beta}$.

Пусть $X \subset \mathcal{T}$. Отображение

$$
d_{X} S(x)=S^{n_{X}(x)}(x), \quad n_{X}(x)=\min \left\{k: k>0, S^{k}(x) \in X\right\}
$$

называется отображением первого возвращения для отображения $S$ и множества $X$. В одномерном случае такие отображения были полностью описаны в [10]. Задача описания отображений первого возвращения для сдвигов многомерного тора крайне далека от своего решения. 
Теорема 9. Пусть

$$
X=\bigsqcup_{j=0}^{d-1} \mathcal{R}_{m, j}(\underbrace{0 \ldots 0}_{m}) .
$$

Тогда существует такое аффинное преобразование $h$, что

$$
d_{X} S=h \circ S \circ h^{-1} .
$$

Теорему 9 можно также переформулировать на языке орбит. Пусть

$$
\text { Orb }=\left\{S^{k}(0)\right\}_{k=-\infty}^{\infty}, \quad \operatorname{Orb}_{X}=\left\{d_{X} S^{k}(0)\right\}_{k=-\infty}^{\infty}
$$

- орбиты отображений $S$ и $d_{X} S$ соответственно. Тогда теорема 9 утверждает, что

$$
h(\mathrm{Orb})=\operatorname{Orb}_{X},
$$

т.е. орбита Orb обладает некоторым свойством самоподобия. Отметим, что, согласно [10], в одномерном случае аналогичное самоподобие возникало только в случае квадратичных иррациональностей (с некоторыми дополнительными ограничениями на разложение в цепную дробь). Таким образом, теорема 9 может быть интерпретирована как некоторый вариант обобщения теоремы Лагранжа о периодичности цепных дробей квадратичных иррациональностей на случай алгебраических иррациональностей произвольной степени.

Перейдем теперь к задаче о времени попадания последовательности дробных долей линейной функции в заданную область. Пусть $\alpha$ иррационально, $I \subset[0 ; 1)$ - некоторый интервал и

$$
n(\alpha, I)=\min \{k: k>0,\{k \alpha\} \in I\} .
$$

В [8] доказан следующий результат.

Теорема 10. Пусть неполные частные разложения $\alpha$ в иепную дробъ ограничены. Тогда

$$
n(\alpha, I)<\frac{C(\alpha)}{|I|} .
$$

В многомерном случае для области $X \subset \mathcal{T}$ естественно определить

$$
n(\beta, X)=\min \left\{k: S^{k}(0) \in X\right\} .
$$

В качестве аналога теоремы 10 естественно было бы ожидать выполнения неравенства

$$
n(\beta, X)<\frac{C(\beta)}{|X|} .
$$

Однако можно показать, что данная оценка неверна ни для какого $C(\beta)$.

Отметим, что величина $n(\beta, X)$ легко оценивается в случае, когда $X$ является множеством ограниченного остатка. Использование множеств $\mathcal{R}_{m, j}(w)$ позволяет доказать следующий результат.

Теорема 11. Пусть $r(X)$ - максимальный радиус $(d-1)$-мерной сферы, которую можно вписать в $X$. Тогда

$$
n(\beta, X)<\frac{C(\beta)}{r^{2}(X)} .
$$

5. Разложения по линейным рекуррентным последовательностям. Перейдем теперь к описанию другого класса теоретико-числовых приложений фракталов и разбиений Рози, связанному с изучением разложений (2) по линейным рекуррентным последовательностям.

Пусть $w \in \mathrm{Adm}$ - допустимое слово, а $\mathbb{N}(w)$ - множество натуральных чисел, для которых слово $\varepsilon_{m(N)}(N) \varepsilon_{m(N)-1}(N) \ldots \varepsilon_{0}(N)$ заканчивается на $w$. Определение множеств $\mathcal{R}_{m, j}$ позволяет доказать следующую теорему.

Теорема 12. Существует такое множество $\mathcal{T}(w)$, что $n \in \mathbb{N}(w)$ тогда и только тогда, когда $S^{n}(0) \in \mathcal{T}(w)$. Множество $\mathcal{T}(w)$ односвязно и представляет собой объединение конечного числа множеств вида $\mathcal{R}_{|w|, j}(w)$. 
Одномерный аналог теоремы 12 впервые появился в [1] и был доказан в максимальной общности в [2]. В этих же работах можно найти многочисленные теоретико-числовые приложения данного результаты и дальнейшие ссылки. Рассмотрим два приложения многомерной теоремы 12.

Пусть $\nu(w)$ - плотность множества $\mathbb{N}(w)$, т.е.

$$
\nu(w)=\lim _{n \rightarrow \infty} \frac{\sharp\{k: 1 \leqslant k \leqslant n, k \in \mathbb{N}\}}{n} .
$$

Внимательный анализ возникающих при доказательстве теоремы 12 разложений множества $\mathcal{T}(w)$ на множества $\mathcal{R}_{|w|, j}(w)$ позволяет доказать следующий результат.

Теорема 13. Множество $\{\nu(w):|w|=m\}$ содержит не более $2^{d-1}$ элементов. При достаточно большой длине слова $ш$ оно содержит в точности $2^{d-1}$ элементов.

Пусть $r_{k}(N)$ - число решений диофантова уравнения

$$
n_{1}+\ldots+n_{k}=N
$$

с дополнительными условиями на слагаемые $n_{j} \in \mathbb{N}(w)$.

Теорема 14. Справедлива асимптотическая формула

$$
r_{k}(N) \sim \frac{1}{(k-1) !|\mathcal{T}|^{k-1}} c_{k}\left(S^{N}(0)\right) N^{k-1},
$$

где

$$
c_{k}(x)=\int_{\mathcal{T}} \chi_{w}\left(x_{1}\right) \chi_{w}\left(x_{2}-x_{1}\right) \ldots \chi_{w}\left(x_{k-1}-x_{k-2}\right) \chi_{w}\left(x-x_{k-1}\right) d x_{1} \ldots d x_{k-1},
$$

a $\chi_{w}(x)$ - характеристическая функиия множества $\mathcal{T}(w)$, продолженная по периодичности относительно решетки $L$.

Аналог теоремы 13 был доказан в [9] для ограничений вида $\left\{n_{j} \alpha\right\} \in I_{j}$, где $I_{j}$ - некоторые интервалы. Доказательство теоремы 13 получается обобщением рассуждений из [9] на случай дробных долей на многомерном торе и применением теоремы 9. Отметим, что аналог теоремы 13 может быть получен и для ограничений вида $n_{j} \in \mathbb{N}\left(w_{j}\right)$ с разными словами $w_{j}$.

Поскольку формула для функции $c_{k}(x)$ из теоремы 13 является достаточно сложной, представляет интерес вопрос о получении оценок данной функции, в частности, о нахождении условий, при которых данная функция всюду положительна. Ответ дается следующими результатами.

Предложение 4. Справедливо неравенство

$$
\left|c_{k}(x)\right| \leqslant|\mathcal{T}(w)|^{k-1} .
$$

Предложение 5. Существует такая постоянная $c(w)>0$, что при

$$
k>\left[\frac{|\mathcal{T}|}{|\mathcal{T}(w)|}\right]+1
$$

справедливо неравенство

$$
\left|c_{k}(x)\right| \geqslant c(w)|\mathcal{T}(w)|^{k-1} .
$$

Отметим также следующий результат.

Предложение 6. Справедливо равенство

$$
\int_{\mathcal{T}} c(x) d x=\frac{|\mathcal{T}|^{k}}{(k-1) !} .
$$


6. Обобщенное круговое умножение Кнута-Матиясевича. Пусть $\left\{F_{n}\right\}$ - последовательность Фибоначчи, задаваемая соотношением $F_{n}=F_{n-1}+F_{n-2}$ и начальными условиями $F_{1}=F_{2}=1$. Любое натуральное число $N$ имеет жадное разложение по последовательности $\left\{F_{n}\right\}$. Данное разложение имеет вид

$$
N=\sum_{n} \varepsilon_{n}(N) F_{n}
$$

где $\varepsilon_{n}(N)$ принимает значение 0 или 1 , причем $\varepsilon_{n}(N) \varepsilon_{n+1}(N)=0$. Ю. В. Матиясевич (см. [7]) ввел понятие кругового умножения Фибоначчи

$$
N \circ M=\sum_{k} \sum_{l} \varepsilon_{k}(N) \varepsilon_{l}(M) F_{k+l} .
$$

Очевидно, введенная операция коммутативна. Также легко проверить, что она не является дистрибутивной относительно сложения. Кнут доказал, что данная операция является ассоциативной (см. [21]).

В случае общего $T$-разложения (2) обобщенное круговое умножение $\circ_{z}$ определяется равенством

$$
N \circ_{z} M=\sum_{k=0}^{m(N)} \sum_{l=0}^{m(M)} \varepsilon_{k}(N) \varepsilon_{l}(M) T_{k+l+z} .
$$

Появление дополнительного аргумента $z$ мотивируется следующей теоремой (см. [16]).

Теорема 15. При достаточно больших z умножение $\circ_{z}$ ассочиативно.

Введем отображение

$$
\Phi_{\mathbb{N}, z}(N)=\Phi\left(\sum_{k=0}^{m(N)} \varepsilon_{k}(N) \beta^{k+z}\right) .
$$

Множество $\mathcal{T}_{z}=\overline{\Phi(\mathbb{N})}$ является аффинным образом множества $\mathcal{T}$ и, следовательно, по теореме 2 является фундаментальной областью некоторой решетки $L_{z}$.

Непосредственные вычисления в сочетании с использованием теоремы 4 дают следующий результат.

Теорема 16. Справедливы равенства

$$
\begin{gathered}
\Phi_{\mathbb{N}, z}(N+M) \equiv \Phi_{\mathbb{N}, z}(N)+\Phi_{\mathbb{N}, z}(M) \quad\left(\bmod L_{z}\right), \\
\Phi_{\mathbb{N}, z}\left(N \circ_{z} M\right) \equiv \Phi_{\mathbb{N}, z}(N) \Phi_{\mathbb{N}, z}(M) \quad\left(\bmod L_{z}\right) .
\end{gathered}
$$

Можно также показать, что разности между правыми и левыми частями равенств в теореме 16 могут принимать только конечное множество значений.

Аналог отображения $\Phi$ впервые был построен В. Г. Журавлевым (см. [4]) в случае, когда $\left\{T_{n}\right\}$ является классической последовательностью Фибоначчи и $z=-1$. В. Г. ЖКуравлев рассматривал аналоги классических диофантовых уравнений первой и второй степени, в которых обычное умножение заменяется круговым умножением Фибоначчи. Теорема 16 также позволяет получать результаты об уравнениях над множеством натуральных чисел с операциями $\left(\circ_{z},+\right)$ на основе результатов об уравнениях в кольце $R=\mathbb{Z}\left[\beta^{(1)}\right] \oplus \ldots \oplus \mathbb{Z}\left[\beta^{\left(r_{1}+r_{2}\right)}\right]$ с дополнительными ограничениями, накладываемыми на решения. Метод позволяет доказывать существование решений таких уравнений, а также получать нижние оценки, а в некоторых случаях и асимптотические формулы для числа решений.

На основе теоермы 16 можно получить следующий результат об ассоциативности и дистрибутивности обобщенных круговых умножений Кнута-Матиясевича.

Теорема 17. Для любого z существует такое подмножество $\mathbb{N}_{z} \subseteq \mathbb{N}$ положительной плотности, что для любых $N_{1}, N_{2}, N_{3} \in \mathbb{N}_{z}$ выполняются равенства

$$
\begin{gathered}
\left(N_{1} \circ_{z} N_{2}\right) \circ_{z} N_{3}=N_{1} \circ_{z}\left(N_{2} \circ_{z} N_{3}\right), \\
\left(N_{1}+N_{2}\right) \circ_{z} N_{3}=N_{1} \circ_{z} N_{3}+N_{2} \circ_{z} N_{3} .
\end{gathered}
$$


Отметим, что если в утверждении теоремы 17 ограничиться только ассоциативностью, то $\mathbb{N}_{z}$ можно выбрать замкнутым относительно умножения ${ }_{z}$.

Для классического кругового умножения Фибоначчи справедлива явная формула

$$
N \circ M=N M+[(N+1) \tau] M+[(M+1) \tau] N,
$$

где [·] - целая часть числа и $\tau=(\sqrt{5}-1) / 2$ (см. [7]). Аналогичная формула для умножения Фибоначчи и $z=-1$ получена в [4]. Использование фракталов Рози позволяет получить формулы для кругового умножения для общих последовательностей $\left\{T_{k}\right\}$ и произвольных $z$. Ограничимся для простоты случаем $z=0$.

Теорема 18. Справедливо равенство

$$
N \circ_{0} M=N M+\sum_{i=1}^{d-1} \sum_{j=1}^{d-1} c_{i j} N\left(\mathcal{R}_{j}, N\right) N\left(\mathcal{R}_{j},(M)\right)
$$

с цельми $c_{i j}$, зависящими от последовательности $\left\{T_{k}\right\}$.

\section{СПИСОК ЛИТЕРАТУРЫ}

1. Давлетлрова Е. П., Жукова А. А., Шутов А. В. Геометризация системы счисления Фибоначчи и ее приложения к теории чисел// Алгебра и анализ. - 2013. - 25, № 6. - С. 1-23.

2. Жукова А. А., Шутов А. В. Геометризация системы счисления// Чебышев. сб. $-2017 .-18$, № 4. C. $221-244$.

3. Журавлев В. Г. Разбиения Рози и множества ограниченного остатка// Зап. науч. семин. ПОМИ. 2005. - 322. - C. 83-106.

4. Журавлев В. Г. Суммы квадратов над о-кольцом Фибоначчи// Зап. науч. семин. ПОМИ. - 2006. 337. - C. $165-190$.

5. Журавлев В. Г. Множества ограниченного остатка// Зап. науч. семин. ПОМИ. $-2016 .-445$. C. $93-174$.

6. Кузнецова Д. В., Шутов А. В. Перекладывающиеся разбиения тора, подстановка Рози и множества ограниченного остатка// Мат. заметки. - 2015. - 98, № 6. - С. 878-897.

7. Матиясевич Ю. В. Связь систем уравнений в словах и длинах с 10-й проблемой Гильберта// Зап. науч. семин. ЛОМИ. - 1968. - 8. - С. 132-144.

8. Шутов А. В. О скорости достижения точных границ остаточного члена в проблеме Гекке-Кестена// Чебышев. сб. - 2013. - 14, № 2. - С. 173-179.

9. Шутов А. В. Об одной аддитивной задаче с дробными долями// Науч. вед. БелГУ. Сер. Мат. Физ. - 2013. - 5 (148), № 30. - С. 111-120.

10. Шутов А. В. Производные поворотов окружности и подобие орбит// Зап. науч. семин. ПОМИ. 2004. - 314. - C. 272-284.

11. Akiyama S. Self affine tiling and Pisot numeration system// in: Number Theory and Its Applications (Gyory K., Kanemitsu S., eds.). — Dordrecht: Kluwer, 1999. - P. 7-17.

12. Akiyama S. On the boundary of self affine tilings generated by Pisot numbers// J. Math. Soc. Jpn. — 2002. - 54, № 2. - P. 83-308.

13. Akiyama S., Barat G., Berthe V., Siegel A. Boundary of central tiles associated with Pisot beta-numeration and purely periodic expansions// Monats. Math. - 2008. - 155. - P. 377-419.

14. Arnoux P., Ito S. Pisot substitutions and Rauzy fractals// Bull. Belg. Math. Soc. Simon Stevin. - 2001. - 8, № 2. - P. 181-207.

15. Berthe V., Siegel A., Thuswaldner J. Substitutions, Rauzy fractals, and tilings// in: Combinatorics, Automata, and Number Theory. — Cambridge Univ. Press, 2010. - P. 248-323.

16. Grabner P. J., Pethõ A., Tichy R. F., Woeginger G. J. Associativity of recurrence multiplication// Appl. Math. Lett. - 1994. - 7, № 4. - P. 85-90.

17. Grepstad S., Lev N. Sets of bounded discrepancy for multi-dimensional irrational rotation// Geom. Funct. Anal. - 2015. - 25, № 1. - P. 87-133.

18. Frougny C., Solomyak B. Finite beta-expansions// Ergod. Theory Dynam. Sys. - 1992. — 12. - P. $713-$ 723. 
19. Hecke E. Über analytische Funktionen und die Verteilung von Zahlen mod. eins// Abhand. Math. Sem. Hamburg Univ. - 1921. - 5, № 1. - P. 54-76.

20. Liardet P. Regularities of distribution// Compos. Math. — 1987. — 61, № 3. - P. 267-293.

21. Knuth D. Fibonacci multiplication// Appl. Math. Lett. - 1988. — 1, № 2. - P. 3-6.

22. Pytheas Fogg N. Substitutions in Dynamics, Arithmetics and Combinatorics. - Springer, 2001.

23. Rauzy G. Nombres algebriques et substitutions// Bull. Soc. Math. France. - 1982. - 110. - P. 147-148.

24. Siegel A., Thuswaldner J. Topological properties of Rauzy fractals. — Soc. Math. France, 2009.

Шутов Антон Владимирович

Владимирский государственный университет им. А. Г. и Н. Г. Столетовых

E-mail: a1981@mail.ru 